\begin{abstract}
000
001

002

003

004

005

006

007

008

009

010

011

012

013

014

015

016

017

018

019

020

021

022

023

024

025

026

027

028

029

030

031

032

033

034

035

036

037

038

039

040

041

Abstract

The fast radial symmetry (FRS) transform has been very popular for detecting interest points based on local radial symmetry ${ }^{1}$. Though, FRS delivers good performance at a relatively low computational cost and is very well suited for a variety of real-time computer vision applications, it is not invariant to perspective distortions. However, even perfectly (radially) symmetric visual patterns in the real world are perceived by us after a perspective projection. In this paper, we propose a systematic extension to the FRS transform to make it invariant to (bounded) cases of perspective projection - we call this transform the generalized FRS or GFRS transform. We show that GFRS inherits the basic characteristics of FRS and retains its computational efficiency. We demonstrate the wide applicability of GFRS by applying it to a variety of natural images to detect radially symmetric patterns that have undergone significant perspective distortions. Subsequently, we build a nucleus detector based on the GFRS transform and apply it to the important problem of digital histopathology. We obtain superior performance over state-of-the-art nuclei detection algorithms, which is validated through quantitative measurement of precision and recall.
\end{abstract}

042

043

044

045

046

047

048

049

050

051

052

053

\section{Introduction}

Symmetry of all kinds, including the visual, is ubiquitous in our world. Indeed, visual symmetry can be copiously seen in nature as well as in human creations: buildings, objects, our works of art. Perception of visual symmetry is thought to play an important biolog-

\footnotetext{
${ }^{1}$ The term radial symmetry is used in the sense of circular symmetry, as in the FRS paper [10].
}

\title{
Fast Radial Symmetry Detection Under Affine Transformations
}

\author{
Anonymous CVPR submission
}

Paper ID 560

ical and evolutionary role in humans as well in other species (for examples, see [5],[7]). Perhaps due to this importance of visual symmetry, humans seem to be able to recover symmetry in shapes and random textures within 100ms [16].

This paper deals with symmetry of the radial kind. Our world abounds in objects and structures that are (partially) radially symmetric - natural objects including the heavenly bodies, fruits and vegetables of various kinds, human heads, textured patterns like the spots on a cheetah, a variety of man-made objects including balls, coins, wheels, tapes, manholes, circular tabletops, ends of cylindrical objects as well as innumerable examples from our works of art. Accordingly, computation of radial symmetry has attracted due interest from the computer vision community (e.g. [14], [10], [9]). For an excellent recent exposition, refer to [12].

However, the issue of perspective projection in fast radial symmetry detection seems to have received lesser attention - one work in this direction is [3]. Humans perceive objects after a perspective projection. It is well known that under (bounded cases) of perspective projection, circles give rise to ellipses. Thus, (roughly) radially symmetric visual patterns would be perceived as elliptical by our eyes. To be able to handle perspective projections, it is very important that radial symmetry detectors principally incorporate detection of elliptical radial symmetry in visual patterns.

We are interested in fast computation of radial symmetries. The best run-time [12] is achieved by the Fast Radial Symmetry (FRS) transform proposed by Loy and Zelinsky [10] in 2003. FRS uses a Hough Transform [1] like voting scheme in the hough space to detect points of local radial symmetry. However, instead of using a 3-dimensional parameter space for voting, it 
estimates the local differential properties of the image to reduce the voting space to become one dimensional (scale/ radii axis). If we are looking to detect circles of a give size, the algorithm is $\mathrm{O}(\mathrm{N})$ - $\mathrm{N}$ being the number of pixels in the image.

We make the following contributions in this paper: (1) We systematically extend the FRS transform to incorporate detection of elliptical symmetry while leveraging the very good complexity behavior of the FRS algorithm. We extend the idea of using local differential structure of images (in FRS) to make it work for a class of affine spatial transformations. The proposed algorithm is parametrized to be able to use FRS as a module and thus retains its attractive features like simplicity and fast computational speed. We call the proposed method - the Generalized Fast Radial Symmetry (GFRS) transform. (2) We demonstrate the wide applicability of GFRS by applying it to a variety of natural images to detect radially symmetric patterns that have undergone significant perspective distortions. (3) Finally, we carefully validate the novel GFRS transform by applying it to the problem of (cell) nuclei detection in histopathology slides. These nuclei are elliptical in nature. For this purpose, we train a simple nucleus detector based on the GFRS transform and show that we are able to obtain superior performance over stateof-the-art nuclei detection algorithms (including one based on the FRS detector). We validate the performance through quantitative measurement of precision and recall.

The rest of the paper is structured as follows: In subsection 1.1, we place this work in context with the state of art in computer vision. In Section 2, we present the Generalized Fast Radial Symmetry (GFRS) transform. We present experimental results on natural images in subsection 3.1, and subsequently, in subsection 3.2, we present the application of the ellipse detector based on the GFRS transform to nuclei detection. We summarize the contributions of the paper in Section 4.

\subsection{Related Work}

An area related to our work is that of affine invariant interest point detectors or (local) feature detectors - for an excellent survey, see [15]. Affine invariant detectors like Harris-Affine and Hessian-Affine are very local in nature though they do respond to blob-like features as well. These detectors only use the very local differen- tial properties of the image. GFRS uses the local differential image properties but agglomerates them using an underlying elliptical model. Even though many of these invariant detectors analyze local second order image properties and output elliptical representations, these are based on a fitting process and not on detection of radial symmetry. Similar is the case with region detectors like MSER [11].

More closely related is the ellipse detection based on Generalized Hough Transforms (GHT) [1]. However, this would imply dense sampling in the five dimensional parameter space $\left(c_{x}, c_{y}, \theta, a, b\right)$ - which can be computationally prohibiting. Indeed, to alleviate this problem, Loy and Zelinski [10] proposed the Fast Radial Symmetry (FRS) based on an efficient voting algorithm. FRS transforms an input image to a transform image which highlights points of high radial symmetry. The FRS algorithm is very efficient with a complexity linear in the size of the image (for each scale). However, FRS is not invariant to affine transformations. Our work addresses precisely this issue. We systematically extend the FRS algorithm to detect radial symmetries while being invariant to affine transformations.

More recently, Cornelius and Loy [3] proposed an approach to detect radial symmetry under affine projections. However, their approach is computationally intensive: they first detect affine invariant features, compute SIFT descriptors and pairs of features are used to vote for. We, on the other hand, provide a natural extension to the FRS transform retaining the computational efficiency of the original algorithm.

\section{Generalized Fast Radial Symmetry (GFRS) Transform}

\subsection{Fast radial symmetry transform}

We summarize here the fast radial symmetry transform method. Interested readers may refer to [10] for more details. For each radius $n$, the algorithm uses image gradients to vote for both the positively-affected and negatively-affected pixels which are defined as

$$
\begin{aligned}
& \mathbf{p}_{+v e}(\mathbf{p})=\mathbf{p}+\text { round }\left(\frac{\mathbf{g}(\mathbf{p})}{\|\mathbf{g}(\mathbf{p})\|} n\right) \\
& \mathbf{p}_{-v e}(\mathbf{p})=\mathbf{p}-\operatorname{round}\left(\frac{\mathbf{g}(\mathbf{p})}{\|\mathbf{g}(\mathbf{p})\|} n\right)
\end{aligned}
$$


$\mathbf{p}_{+v e}(\mathbf{p}), \mathbf{p}_{-v e}(\mathbf{p})$ correspond to pixels with the gradient $\mathbf{g}(\mathbf{p})$ pointing towards and away from the center respectively. From those pixels, an orientation projection image $O_{n}$ and a magnitude projection image $M_{n}$ are formed. Specifically, for each positively affected pixel, the corresponding point $\mathbf{p}_{+v e}$ in $\mathbf{O}_{n}$ and $\mathbf{M}_{n}$ is increased by 1 and $\|\mathbf{g}(\mathbf{p})\|$, respectively. Similarly, for the negatively affected pixel, the corresponding point is decreased by the same quantity in each image.

Then the radial symmetry response map is defined as

$$
S_{n}=F_{n} * A_{n}
$$

where

$$
\begin{gathered}
F_{n}(\mathbf{p})=\frac{M_{n}(\mathbf{p})}{k_{n}}\left(\frac{\left|\tilde{O}_{n}(\mathbf{p})\right|}{k_{n}}\right)^{\alpha} \\
\tilde{O}_{n}(\mathbf{p})=\left\{\begin{array}{cc}
O_{n}(\mathbf{p}), & O_{n}(\mathbf{p})<k_{n} \\
k_{n}, & \text { otherwise }
\end{array}\right.
\end{gathered}
$$

$A_{n}$ is an isotropic Gaussian smoothing function, $\alpha$ is the radial strictness parameter, and $k_{n}$ is a scaling factor across different radii.

While [10] is very effective at detecting circular radial symmetry, FRS is not invariant to spatial transformations arising due to perspective projections. In those cases the gradient direction deviates from the radial vector, which leads to diffusion and dispersion of the locus of symmetry in the object space [17]. In the case of bounded perspective projection, it is well known that circular structures project as elliptical structures. Thus, to handle geometric distortions due to perspective projections, there is a need to extend FRS to handle elliptical symmetries. We use here a simple observation from geometry: an ellipse can be represented as an affine transform of a unit circle. Utilizing this affine relationship, we propose to get a modified voting procedure to generalize the FRS algorithm to render it invariant to (bounded) perspective transformations. We describe the details below.

\subsection{Generalized radial symmetry voting}

Let $p(\phi)$ be the parametrization of a circle

$$
p(\phi)=\left(\cos (\phi)+c_{x}, \sin (\phi)+c_{y}\right)^{T}, 0 \leq \phi<2 \pi
$$

where $c=\left(c_{x}, c_{y}\right)^{T}$ is the center of the circle. Then, the ellipse $q(\phi)$ at the same location $c$, with orientation $\theta$, and $(a, b)$ as the length of the semi-major and semiminor axes respectively, can be obtained by a suitable affine transformation $G$

$$
\begin{gathered}
q(\phi)=G \cdot(p(\phi)-c)+c, G=R \cdot S \\
R=\left(\begin{array}{cc}
\cos (\theta) & -\sin (\theta) \\
\sin (\theta) & \cos (\theta)
\end{array}\right), S=\left(\begin{array}{ll}
a & 0 \\
0 & b
\end{array}\right),
\end{gathered}
$$

where $R, S$ are the rotation and scaling matrix respectively. We denote $A(2)$ as the group of affine transformation of the plane [6], and we restrict $G$ to be a member of $A(2)$ to ensure the uniqueness of the affine transformation [13].

Let us also represent the corresponding tangent vectors as $T_{p(\phi)}, T_{q(\phi)}$ and normal vectors as $N_{p(\phi)}, N_{q(\phi)}$ at $p(\phi)$ and $q(\phi)$. We derive two propositions following from the simple fact that since $G: R^{2} \rightarrow R^{2}$ is a linear transform, the relevant tangent and normal subspaces are also related by the same transformation $G$.

Proposition 1. The tangent vector $T_{q(\phi)}$ at the point $q(\phi)$ on the ellipse can be obtained through the affine transform $G$ of the tangent vector $T_{p(\phi)}$ at the corresponding point $p(\phi)$ on the circle, i.e., $T_{q(\phi)}=$ $G \cdot T_{p(\phi)} \cdot$

Proof. Follows from differentiating (5): $T_{q(\phi)}=$ $\partial_{\phi} q(\phi)=\partial_{\phi} G \cdot p(\phi)=G \cdot T_{p(\phi)}$

Proposition 2. The voting vector $V_{q(\phi)}$ toward the centroid of the ellipse at point $q(\phi)$ can be obtained through the affine transform $G$ of the normal vector $N_{p(\phi)}$ of the corresponding point $p(\phi)$ on the circle, i.e., $V_{q(\phi)}=G \cdot N_{p(\phi)}$.

Proof. Since $V_{q(\phi)}=c-q(\phi)$ and $N_{p(\phi)}=c-p(\phi)$, the result follows from (5).

This leads to the main result of our paper.

Proposition 3. Let $\hat{T}_{q(\phi)}$ be an unbiased estimator of the tangent at $q(\phi)$. Then, the unbiased estimator of the voting direction is given by

$$
\hat{V}_{q(\phi)}=G \cdot M \cdot G^{-1} \cdot \hat{T}_{q(\phi)}
$$

where $M=\left[\begin{array}{cc}0 & 1 \\ -1 & 0\end{array}\right]$. 


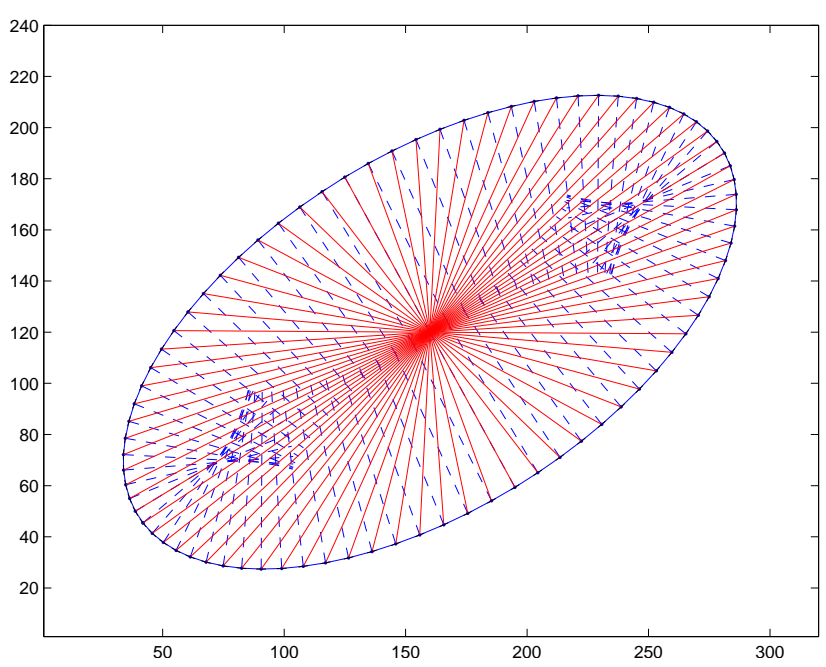

Figure 1. Voting direction $V_{q(\phi)}$ for the ellipse's centroid (in red) vs. Original normal vectors $N_{q(\phi)}$ on the ellipse (in blue).

Proof. $V_{q(\phi)}=G \cdot N_{p(\phi)}=G \cdot M \cdot T_{p(\phi)}=G \cdot M$. $G^{-1} \cdot T_{q(\phi)}$.

The first equality follows from Proposition 2, the second from the fact that $\mathrm{M}$ relates the tangent and normal spaces and the third from Proposition 1.

In Figure 1, we illustrate that the voting vectors $\hat{V}_{q(\phi)}$ (red lines), as computed by (6) have the correct directions as they all correctly intersect at the center of the ellipse. On the other hand, the voting directions of FRS, aligned with the normal to the curve, are incorrect. These are depicted as dashed blue lines.

Consequently, for a given $G$, we can use local differential image characteristics to vote for the ellipses of that particular shape. Therefore, for each point sampled from the constrained affine group $A(2)$, we modify the image gradient voting vector in [10] to (6). Then, each value of $G$ provides us with a response map, which captures evidence for ellipses of that particular size, shape, and orientation. Note that $G$ induces a natural parametrization for the space of ellipses and a consequent generalization of the FRS algorithm (GFRS). We can now span the desired range of the 3D parameter space of $A(2)$, compute the GFRS map for each parameter and use the resultant stack of response maps as an interest point map for ellipses of all desired sizes, shapes and orientations. The final response map is obtained through taking the maximum at each pixel location among all the response maps.
We summarize the detailed procedures of GFRS in Algorithm 1.

In the following, we summarize two practical aspects.

Normalizing factor $k_{n}$ : As the length of the major/minor axis of ellipse changes, so does the number of gradient votes from the perimeter of the ellipse. To alleviate this bias, we follow the suggestion in [10], and empirically determine the normalizing factor $k_{n}$ (see 2) for ellipse across different affine transformation parameters $a$ and $b$.

Smoothing: We generalize the smoothing performed in (1) to be consistent with the affine transformation by choosing a multivariate Gaussian kernel, the orientation and extend is determined by the affine parameters $\theta, a$, and $b$. This is motivated by the observation that, under noise, the deviation of the voting location from the true center is increasing with the length of $V_{q(\phi)}$.

\section{Experimental results}

In this section, we apply GFRS transform to a variety of real world images and nuclei detection in histopathological images. We provide the details below.

\subsection{Real world images}

We apply GFRS to a variety of complex real world images to detect regions of radial symmetry in presence of significant perspective distortion. The examples we present contain wheels from different kinds of vehicles, as well as other objects such as tomatoes and coins. In Figure 2, we show the response map from GFRS in the top row and the corresponding image overlaid with detections in the bottom row. We observe that the GFRS response maps capture locations of radially symmetric structures very effectively, despite perspective transformations and other challenging conditions, e.g, cluttered background, partial occlusion of objects' elliptical-arcs, lack of contrast in regions of interest etc. The high response regions coincide well with locations of radially symmetric objects like wheels, coins etc. Note that the range of scales of the sampling points from $A(2)$ is chosen not to target very small objects so as not to clutter the qualitative results. We observe the overlaid ellipses coincide well with the boundaries of the radially symmetric ob- 
Algorithm 1 Generalized fast radial symmetry transform.

Require: Image gradient $\mathrm{g}(\mathrm{p})$ at each pixel $\mathrm{p}$, a set of sampling points from affine transform subspace $\left\{G_{i}\right\}$

1: Get the tangent vector $T_{p}$ at each pixel $p$, which is perpendicular to the gradient vector $\mathrm{g}(\mathrm{p})$.

2: For each sampled affine transformation $G_{i}$, modify the image gradient voting vector in [10] to (6) at each image pixel $p$, collect the corresponding response map $F_{i}$.

3: Take the maximum at each pixel from all response maps $\left\{F_{i}\right\}$ as the GFRS response map. Thresholding the response map and do a non-maxima suppression to obtain detected elliptical regions.

jects. This also shows that GFRS is able to estimate the affine parameters quite accurately.

For comparison, we applied FRS to some images of Figure 2. The results are shown in Figure 3. As expected, GFRS is able to detect radial symmetry in the presence of significant perspective distortions while FRS fails to do so.

\subsection{Nuclei detection}

We now access the performance of GFRS for detecting nuclei in biopsy samples from histopathological images. Nuclei detection is a fundamental step in the automatic prognosis of breast cancer. Different methods have been proposed in previous work, including a linear SVM approach [8], a Hessian matrix based approach [2], a circular Hough transform based approach [4] etc. Here, we exploit a-prior knowledge that nuclei are usually elliptical in shape, and apply the GFRS transform to extract those elliptical regions of interest. The samplings from the $3 \mathrm{~d}$ affine space used here are: $a=[6,8,10,12,14,16], b=[4$, $6,8], \theta=\left[i^{*} \pi / 8, \mathrm{i}=0,1, \ldots, 7\right]$. Then from the output response map, we collect all interest points above a certain threshold value. Each interest point is associated with a confidence value and an elliptical region which is described by five ellipse parameters.

We carry out experiments on nuclei detection using $512 \times 512$ image patches that were taken from large, several GPixel large, H\&E stained "virtual slides". Those slides were sampled at 0.47 microns/pixel, corresponding to $40 \mathrm{X}$ objective scan.

Figure 4 shows the nuclei detection results from a typical histopathological image. We show the response map on the left, and the extracted nuclei on the right. We plot ellipses on top of the detected nuclei, and the color of the ellipses represents the confidence value. We observe that GFRS can effectively detect nuclei with various shapes, even in challenging cases of touching/overlapping nuclei. In addition to identifying the nuclei centers, GFRS provides additional information about the size and orientation of the nuclei from the extracted elliptical region. This side information can be used to facilitate follow-up processing and analysis for segmentation, linking, and detection of other conditions like malignancy.

We also carried out a quantitative evaluation and comparison of the GFRS based nuclei detector. For this purpose, we constructed a ground truth dataset by manually annotating all nuclei centroids from five images of size $512 \times 512$, - in all, 2555 nuclei were annotated. We then applied GFRS and evaluated its performance using precision-recall curves.

We compare our results with those from related state of the art - radial symmetry transform [10], Hessian matrix based detection [2], SVM based detection [8]. We plot the precision and recall curves in Figure 5.

GFRS clearly achieves the best performance among all the algorithms that were tested on this problem. For example, for a recall of $95 \%$, only every 13 th detection corresponds to a false alarm. None of the other approaches achieves comparable performance.

\subsection{Computational complexity}

In our experiments, it takes around $20 \mathrm{~ms}$ for each sampled $G_{i}$ to generate a response map on a $375 \times 250$ image. The number of parameters sampled from $3 \mathrm{~d}$ affine space for each image varies from 50 to 200 . However, with application-specific priors, e.g., rough knowledge of camera height and viewing angle in a surveillance/traffic-like static camera setting, GFRS will collapse to almost the same sampling set size as FRS (1d scale space). 


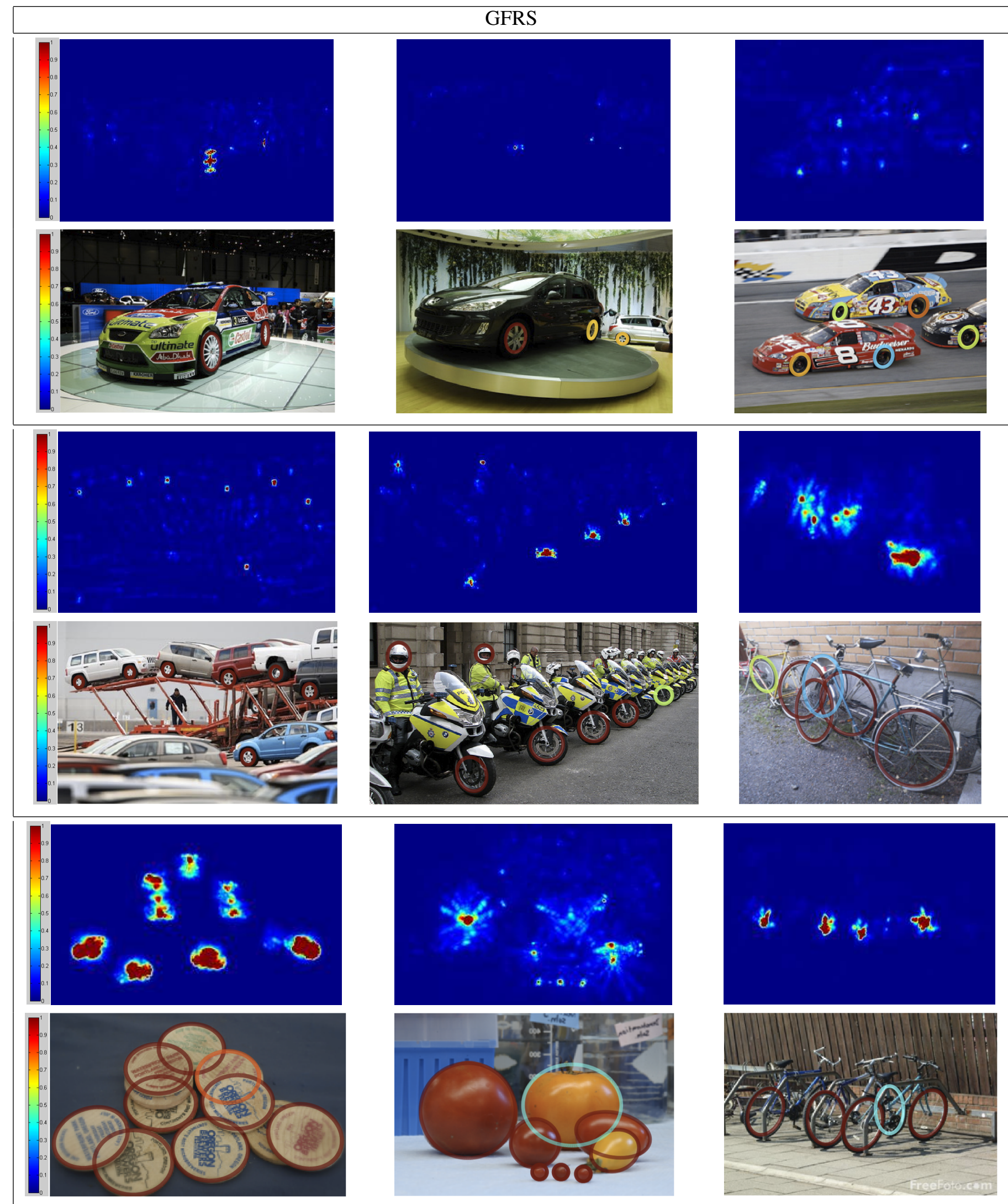

Figure 2. Results of the GFRS applied to different real world images. Each pair of images shows the GFRS response map

and the most confident detections corresponding to ellipses after thresholding the GFRS response map and non-maxima suppression. 


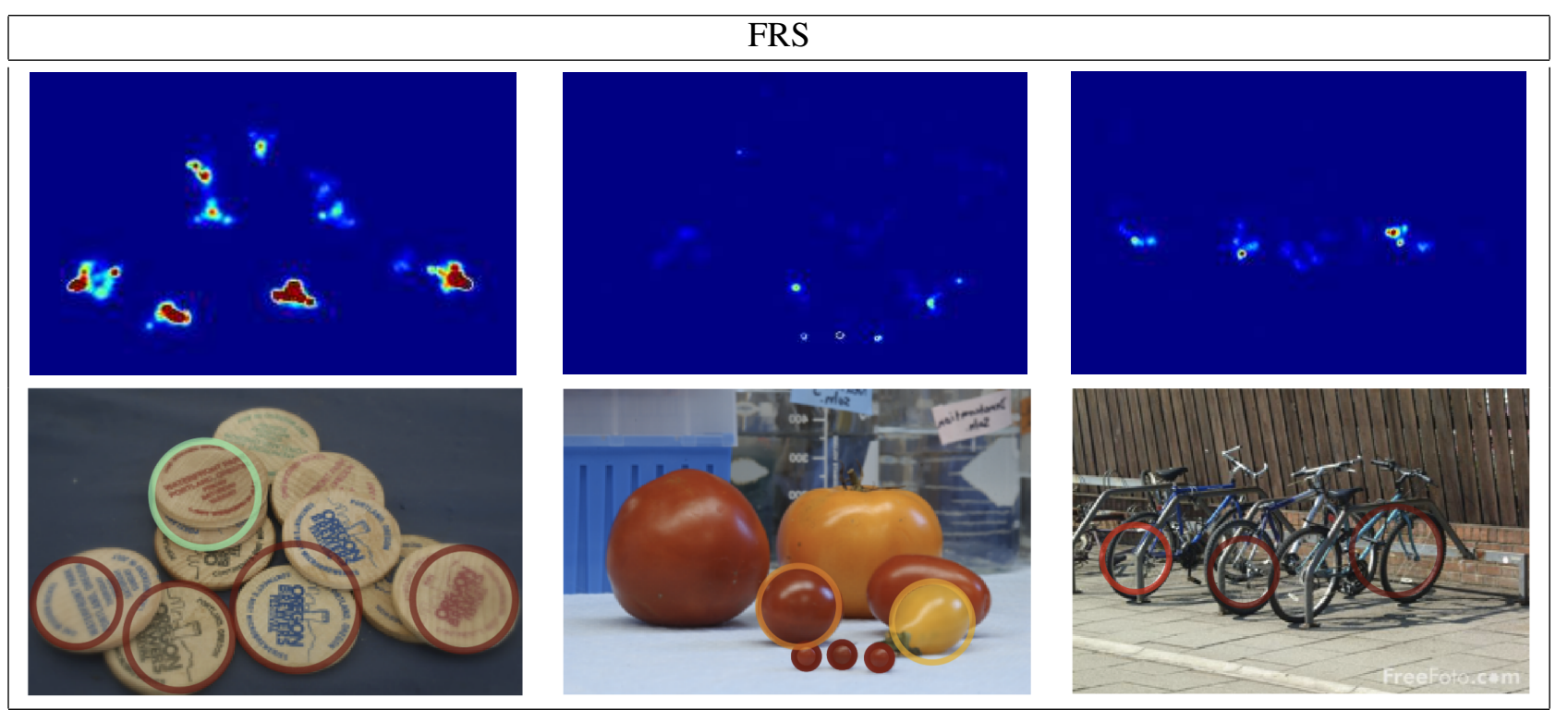

Figure 3. FRS applied to different real world images. Compared to Figure 2, it can be seen that many of the (elliptical) structures are less pronounced.
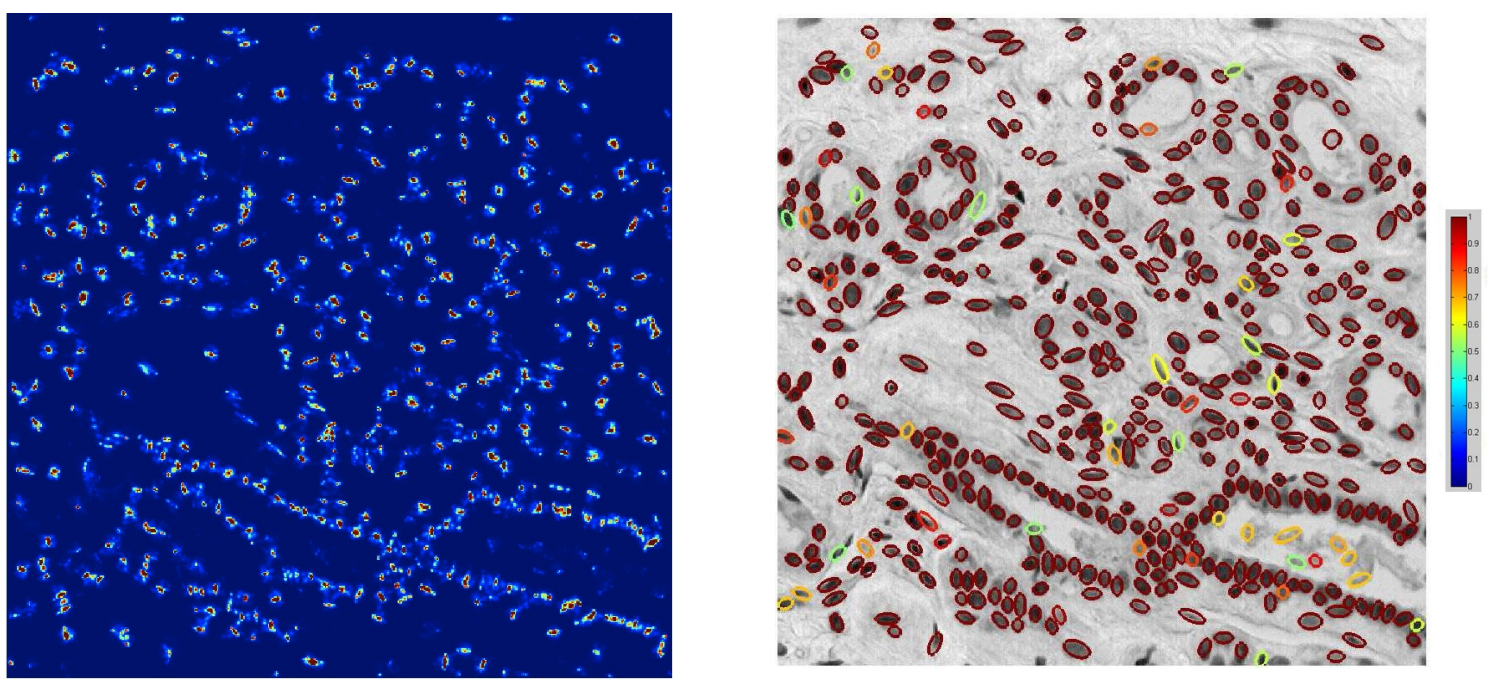

Figure 4. Nuclei detection of one histopathological image. Left: response map from GFRS; Right: detected nuclei.

\section{Conclusions}

In this paper, we have presented a novel GFRS transform that can detect radial symmetry in presence of (bounded) perspective transformations. This was achieved by systematically modifying the FRS transform, which is currently the fastest algorithm for detecting circular symmetry, to detect elliptical symmetry as well. We showed the wide applicability of GFRS to computer vision by applying it to a variety of everyday images. Further, we applied GFRS to the important task of cell nuclei detection in biopsy sam- ples from histopathology images. This is an important first step in automatic determination of presence and malignancy of cancer. We compared the GFRS nucleus detector with other state of the art detectors using precision-recall curves and showed that GFRS achieves the best results reported to date.

\section{Acknowledgement}

Part of this work was supported by National Institutes of Health/National Institute of Biomedical Imaging and Bioengineering (NIH/NIBIB) under Grant 


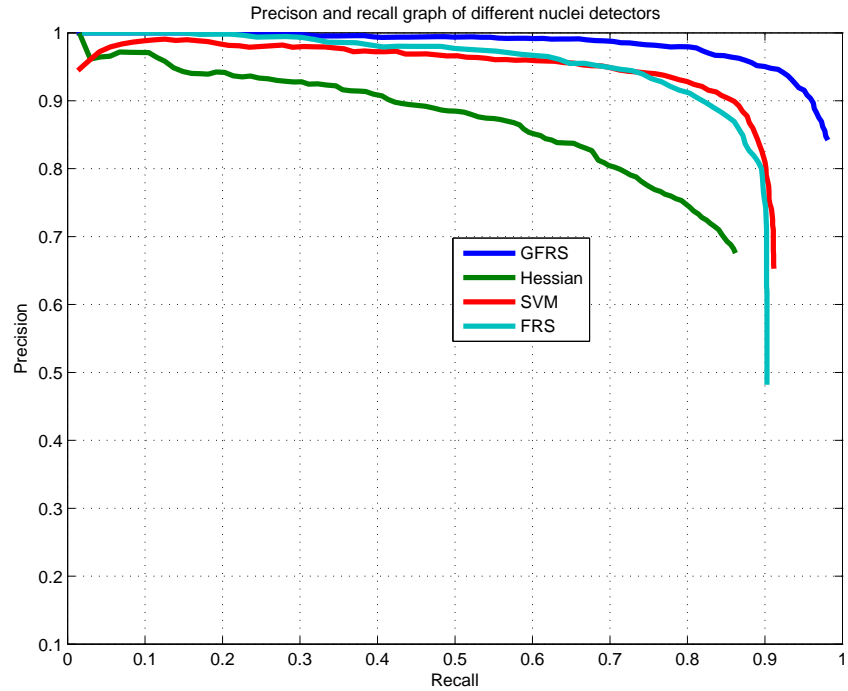

Figure 5. Precision and recall curve of nuclei detection from different methods. GFRS outperforms Hessian, SVM, and FRS based methods significantly.

\section{R01EB008055.}

\section{References}

[1] D. H. Ballard. Generalizing the Hough transform to detect arbitrary shapes. Pattern Recognition, 13:111-122, 1981. 1, 2

[2] C. C. Bilgin, P. Bullough, G. E. Plopper, and B. Yener. Ecm-aware cell-graph mining for bone tissue modeling and classification. Journal of Data Mining and Knowledge Discovery, 2010. 5

[3] H. Cornelius and G. Loy. Detecting rotational symmetry under affine projection. In ICPR, pages 292-295, 2006. 1, 2

[4] E. Cosatto, M. Miller, H. P. Graf, and J. Meyer. Grading nuclear pleomorphism on histological micrographs. In $I C P R$, pages $1-4,2008.5$

[5] K. Grammer and R. Thornhill. Human (homo sapiens) facial attractiveness and sexual selection: The role of symmetry and averageness. $J$. Comp. Psychol., 108:233-242, 1994. 1

[6] Richard Hartley and Andrew Zisserman. Multiple View Geometry in Computer Vision. Cambridge University Press, New York, NY, USA, 2 edition, 2003. 3
[7] G. A. Horridge. The honeybee (apis mellifera) detects bilateral symmetry and discriminates its axis. Journal of Insect Physiology, 42:755-764, 1996. 1

[8] P. Khurd, L. Grady, A. Kamen, S. Gibbs-Strauss, E. Genega, and J. V. Frangioni. Network cycle features: Application to computer-aided gleason grading of prostate cancer histopathological images. In International Symposium on Biomedial Imaging, pages 1632-1636, 2011. 5

[9] S. Lee and Y. Liu. Skewed rotation symmetry group detection. PAMI, 32(9):1659-1672, 2010. 1

[10] G. Loy and A. Zelinsky. Fast radial symmetry for detecting points of interest. PAMI, 25:959-973, August 2003. 1, 2, 3, 4, 5

[11] J. Matas, O. Chum, M. Urban, and T. Pajdlaa. Robust wide-baseline stereo from maximally stable extremal regions. Image and Vision Computing, 22(10):761-767, 2004. 2

[12] J. Maver. Self-similarity and points of interest. PAMI, 32(7):1211-1226, 2010. 1

[13] S. J. Maybank. Application of the Fisher-Rao metric to ellipse detection. Int. J. Comput. Vision, 72:287-307, May 2007. 3

[14] D. Reisfeld, H. Wolfson, and Y. Yeshurun. Context free attentional operators: The generalized symmetry transform. Intl J. Computer Vision, 14:119-130, 1995. 1

[15] T. Tuytelaars and K. Mikolajczyk. Local invariant feature detectors: a survey. Foundations and Trends in Computer Graphics and Vision, 3(3):177-280, 2008. 2

[16] H. R. Wilson and F. Wilkinson. Symmetry perception: A novel approach for biological shapes. Vision Research, 42:589-597, 2002. 1

[17] Q. Yang and B. Parvin. Perceptual organization of radial symmetries. In In Proceedings, Computer Vision and Pattern Recognition, pages 320-325, 2004. 3 